\title{
MODELLING SALIENCY AWARENESS FOR OBJECTIVE VIDEO QUALITY ASSESSMENT
}

\author{
Ulrich Engelke ${ }^{\dagger}$, Marcus Barkowsky*, Patrick Le Callet*, and Hans-Jürgen Zepernick ${ }^{\dagger}$ \\ ${ }^{\dagger}$ Blekinge Institute of Technology, PO Box 520, 37225 Ronneby, Sweden \\ *IRCCyN UMR no 6597 CNRS, Ecole Polytechnique de l'Universite de Nantes, \\ rue Christian Pauc, La Chantrerie, 44306 Nantes, France
}

\begin{abstract}
Existing video quality metrics do usually not take into consideration that spatial regions in video frames are of varying saliency and thus, differently attract the viewer's attention. This paper proposes a model of saliency awareness to complement existing video quality metrics, with the aim to improve the agreement of objectively predicted quality with subjectively rated quality. For this purpose, we conducted a subjective experiment in which human observers rated the annoyance of videos with transmission distortions appearing either in a salient region or in a non-salient region. The mean opinion scores confirm that distortions in salient regions are perceived much more annoying. It is shown that application of the saliency awareness model to two video quality metrics considerably improves their quality prediction performance.
\end{abstract}

Index Terms - Video quality metrics, visual saliency, eye tracking, subjective quality experiment.

\section{INTRODUCTION}

Advanced communication networks facilitate the transition from traditional voice services to multimedia services, including packet based video streaming over IP. This is also enabled through contemporary video coding standards, such as H.264/AVC [1], which allows for encoding at significantly lower bit rates than its predecessors, while maintaining the level of visual quality. However, the limited bandwidths in the networks and the strong signal compression result in the video content being highly prone to visual distortions through bit errors and packet loss during transmission. For service providers it is of vital importance to objectively measure the perceptual impact of these distortions to provide a certain level of Quality of Experience (QoE) to the end user. For this reason there has been an increased effort in recent years to develop objective image and video quality measures.

Earlier visual quality metrics did not take into account that visual content usually exhibits regions of varying saliency, drawing the viewer's visual attention (VA) to different degrees. More recently, however, there have been several studies in which spatial saliency has been incorporated into still image quality metrics to improve quality prediction performance [2-5]. However, there has been only little efforts to incorporate visual saliency into video quality metrics (VQM). This is somewhat surprising, as the effect of saliency driven attention [6] on the perception of distortions is expected to be particularly high in video signals, where the visual scene changes continuously, unlike with still images, where the visual scene is static. In [7] and [8] objective saliency maps have been used in a pooling stage of video quality metrics. Both works rely on a possibly inaccurate prediction of saliency by an objective model and no qualitative or quantitative analysis has been provided regarding the impact of saliency on the perceived annoyance of localised distortions.

In this paper, we aim to improve the quality prediction performance of a contemporary VQM, the temporal trajectory aware video quality measure (TetraVQM) [9], and the well known peak signal-to-noise ratio (PSNR) [10], by adding a simple saliency model. For this purpose, we used eye tracking data from a task-free experiment as a ground truth to identify saliency in a number of videos. We then introduced packet loss into the bit stream of the videos to create localised distortions appearing either in a salient region or a non-salient region. In a second subjective experiment, a number of observers was then asked to rate the annoyance of the distortions in the videos. The mean opinion scores (MOS) show that the annoyance of the distortions depends indeed strongly on the saliency of the region that they appear in. Having this knowledge, we develop a model of saliency awareness and evaluate its effectiveness by applying it to TetraVQM and PSNR, with the aim to improve the agreement of the objectively predicted quality with the MOS from the subjective experiment.

This paper is organised as follows. Section 2 explains the subjective experiment that we conducted. Section 3 reviews the video quality metric TetraVQM. The saliency awareness modelling is presented in Section 4. A performance evaluation of the saliency aware metrics is discussed in Section 5. Conclusions are finally drawn in Section 6.

\section{SUBJECTIVE EXPERIMENT}

We conducted a subjective experiment at the University of Nantes, France, to obtain a ground truth for the perceived annoyance of packet loss distortions. The experiment is discussed in detail in [11] and is summarised in the following. 


\subsection{Creation of distorted test sequences}

We considered 30 reference videos in standard definition (SD) format, provided by the Video Quality Experts Group (VQEG) [12], of which we selected 20 sequences for the experiment with respect to the saliency and the spatial and temporal characteristics of the content. The latter were quantified using spatial information (SI) and temporal information (TI) indicators [13]. The saliency in each sequence was identified using gaze patterns from an earlier eye tracking experiment [14] where the 30 sequences were presented to 37 participants under task free condition. The gaze patterns of all observers were post-processed into saliency maps. An example of a reference sequence frame and an overlayed saliency map is shown in Fig. 1(a) and Fig. 1(b), respectively.

We encoded the sequences in H.264/AVC format [1] using the JM 16.1 reference software [15]. The sequences were encoded with a constant quantization parameter $\mathrm{QP}=28$ and in High profile with an IBBPBBP... GOP structure of two different lengths; 30 frames (GOP30) and 10 frames (GOP10). The frame rate was set to 25 and thus, the GOP lengths correspond to $1.2 \mathrm{sec}$ and $0.4 \mathrm{sec}$, respectively. All sequences were shortened to 150 frames, corresponding to $6 \mathrm{sec}$ duration.

We adapted the Joint Video Team (JVT) loss simulator [16] to introduce packet loss into the H.264/AVC bit stream. The packet loss was introduced into a single I frame in each sequence resulting in error propagation until the next I frame, due to the inter-frame prediction of the $\mathrm{P}$ and $\mathrm{B}$ frames. Thus, the two GOP lengths, $30 / 10$ frames, relate to the maximum error propagation lengths $1.2 / 0.4 \mathrm{sec}$. To have better control regarding the location and extent of the loss patterns we chose a fixed number of 45 macro blocks (MB) per slice. Given that SD video has a resolution of $720 \times 576$ pixels, corresponding to $45 \times 36 \mathrm{MB}$, each slice represents exactly one row of MB.

We introduced packet loss into the reference sequences, $\mathrm{SEQ}_{R}$, such as that the distortions appear either in a salient region or non-salient region. In particular, we created distorted sequences with packet loss introduced in 5 consecutive slices centered around a highly salient region of an I frame. We then created a corresponding sequence with 5 consecutive lost slices introduced into a non-salient region of the same I frame. We created such two sequences for both the GOP30 and GOP10 coded sequences. The subsets of distorted sequences are in the following referred to as $\mathrm{SEQ}_{S, 0.4}$, $\mathrm{SEQ}_{N, 0.4}, \mathrm{SEQ}_{S, 1.2}$, and $\mathrm{SEQ}_{N, 1.2}$, where 0.4 relates to error propagation length for GOP10 and, accordingly, 1.2 relates to GOP30. The indices $S$ and $N$, respectively, denote the salient and the non-salient region. An example of a frame containing distortions in the salient region and in the non-salient region is shown in Fig. 1(c) and Fig. 1(d), respectively.

\subsection{Experiment details}

The experiment was designed according to ITU Rec. BT.500 [17]. The videos were presented on a LVM-401W full HD

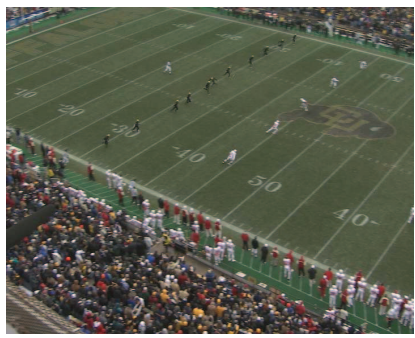

(a)

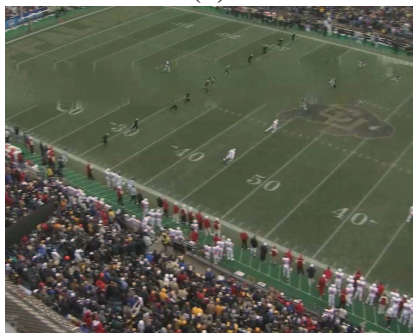

(c)

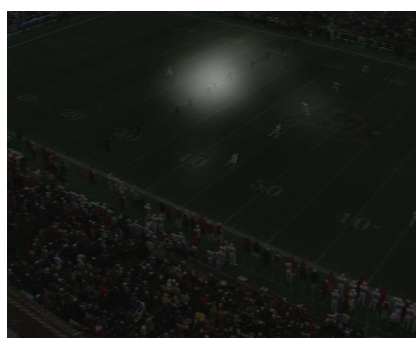

(b)

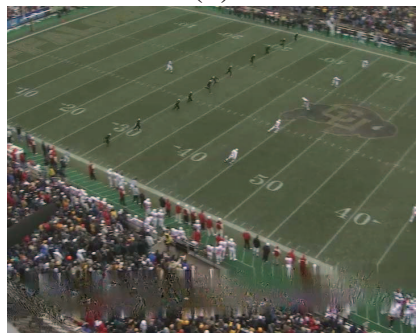

(d)
Fig. 1. Frame 136 of the video sequence 'Kickoff': (a) reference video, (b) saliency information, (c) distortions in the salient region, (d) distortions in the non-salient region.

screen by TVlogic with a size of $40 "$ and a native resolution of $1920 \times 1080$ pixels. A mid-grey background was added to the SD test sequences to be displayed on the HD screen. The observers were seated at a distance of about $150 \mathrm{~cm}$ corresponding to six times the height of the used display area.

Thirty non-expert observers participated in the experiment (10 female, 20 male) with an average age of about 23 years. Prior to each experiment, the visual accuracy of the participants was tested using a Snellen chart and colour deficiencies were ruled out using the Ishihara test. The participants were presented the 100 test sequences ( 20 reference, 80 distorted) in a pseudo random order with a distance between the same content of at least 5 presentations. The sequences were presented using a single stimulus method.

The 5-point impairment scale [17] was used to assess the annoyance of the distortions in the sequences. Here, the observers assigned one of the following adjectival ratings to each of the sequences: 'Imperceptible (5)', 'Perceptible, but not annoying (4)', 'Slightly annoying (3)', 'Annoying (2)', and 'Very annoying (1)'. The impairment scale was given the preference over the quality scale (also defined in [17]), as the rating 'Imperceptible' directly allows to identify whether or not participants actually detected the distortions. The participants were shown 6 training sequences to get a feeling for the distortions to be expected in the test sequences.

\subsection{Experiment outcomes}

The 30 subjective scores for each sequence are averaged into MOS. Corresponding to the subsets of sequences we define MOS subsets $\operatorname{MOS}_{R}, \operatorname{MOS}_{S, 0.4}, \operatorname{MOS}_{N, 0.4}, \operatorname{MOS}_{S, 1.2}$, and 


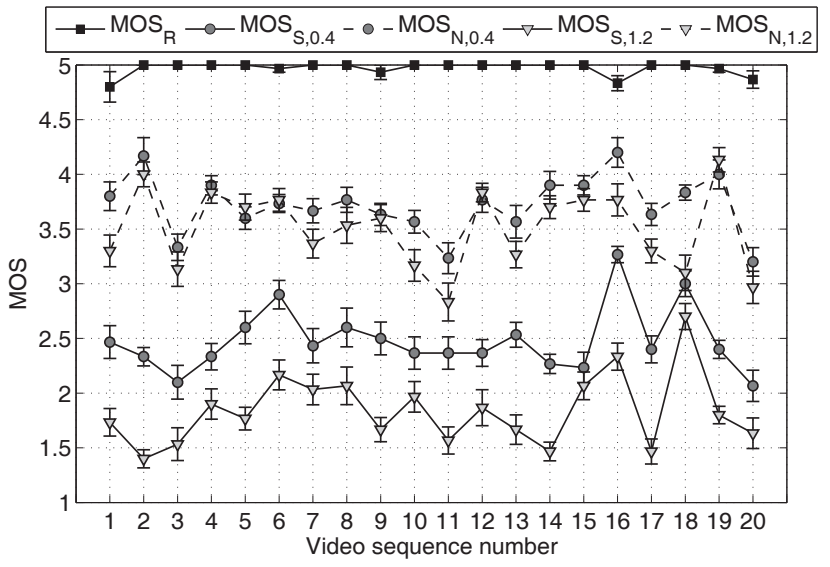

Fig. 2. MOS and standard errors for all 20 sequence contents in each subset.

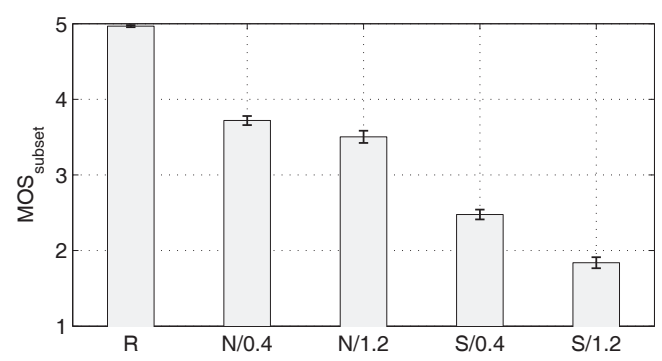

Fig. 3. MOS averaged over all 20 sequences for each subset.

$\mathrm{MOS}_{N, 1.2}$. The MOS subsets, including the standard errors, are shown in Fig. 2 for all 20 contents. It can be observed that the hierarchy of MOS between the subsets is almost exclusively the same for all sequences. The reference sequences $\mathrm{SEQ}_{R}$ always received the highest scores, followed by $\mathrm{SEQ}_{N, 0.4}, \mathrm{SEQ}_{N, 1.2}, \mathrm{SEQ}_{S, 0.4}$, and $\mathrm{SEQ}_{S, 1.2}$.

The MOS for each of the subsets, averaged over all 20 contents, is shown in Fig. 3. It can be seen that the MOS difference between sequences with distortions in the salient region and in the non-salient region is significantly larger as compared to the MOS difference between sequences with long and short distortions. This indicates that the observers distinguish annoyance levels more pronounced with respect to the saliency of the distortion region as compared to the distortion duration. It is particularly worth noting that $S_{E Q, 1.2}$ received an average MOS that is 1.02 higher than for SEQ $S, 0.4$, even though the distortion in the non-salient region is three times longer than the distortion in the salient region.

In summary, the MOS show strong evidence that the visual saliency plays a vital role in judging the perceived annoyance of packet loss degradations. As such, one can expect an improvement in quality prediction performance by incorporating saliency information into VQM.

\section{TETRAVQM}

TetraVQM is an objective quality algorithm that is particularly well suited for the enhancement with visual saliency because it already contains several steps that are motivated by the human visual system. It has been designed for the prediction of video quality in multimedia scenarios including the typical artifacts that occur in packet loss situations.

The TetraVQM algorithm uses the reference and the degraded video to predict the perceived quality. Its main focus is on temporal issues, e.g. the misalignment of the two video sequences, frame freezes and skips, frame rate reduction, influence of scene cuts, and, in particular, the tracking of the visibility of distorted objects. The processing starts with the spatial, temporal, and color alignment of the two input videos. For each aligned image, a spatial distortion map is created which identifies the position and the severity of the degradations using the simple mean squared error (MSE). A human observer perceives the video sequence as a continuous stream of information rather than image by image. For example, it was shown that the perceived severity of artifacts depends on the duration that the artifact is seen. Short distortions are less annoying than longer distortions. However, degradations which move together with an object are perceived as long lasting object degradations rather than several isolated momentary points of distortions. Therefore, TetraVQM estimates the object motion and keeps track of the degradations over time. Each initial distortion map is then modified to account for the temporal visibility of the artifacts.

The spatial summation is performed by applying a filter that is based on the distribution of the cones in the fovea. Currently, the assumption is used that the viewer focuses on the point of the maximum perceived degradation. This was previously seen as the focal point of the observer. Thus, it is straightforward to improve the algorithm by applying a more sophisticated approach that uses the visual saliency. In this paper a first step towards this goal is presented.

A scatter plot of TetraVQM versus the MOS from the experiment we conducted is presented in Fig. 4. In this figure, the sequences corresponding to the four different subsets of distortions $\left(\mathrm{SEQ}_{S, 0.4}, \mathrm{SEQ}_{N, 0.4}, \mathrm{SEQ}_{S, 1.2}, \mathrm{SEQ}_{N, 1.2}\right)$ are illustrated using different markers. In addition, cluster means $\left(\mu_{S, 0.4}, \mu_{N, 0.4}, \mu_{S, 1.2}, \mu_{N, 1.2}\right)$ are provided for all subsets. The scatter plot highlights that TetraVQM accounts for the temporal duration of the distortions but not for the saliency of the distortion region. The latter is evident given the big gap in MOS and the small gap in TetraVQM between the sequences with distortions in the salient and non-salient region.

\section{SALIENCY AWARENESS}

An overview of the saliency awareness model applied to a VQM is depicted in Fig. 5. The idea is to extend a traditional VQM (white blocks) with the saliency awareness model (grey 


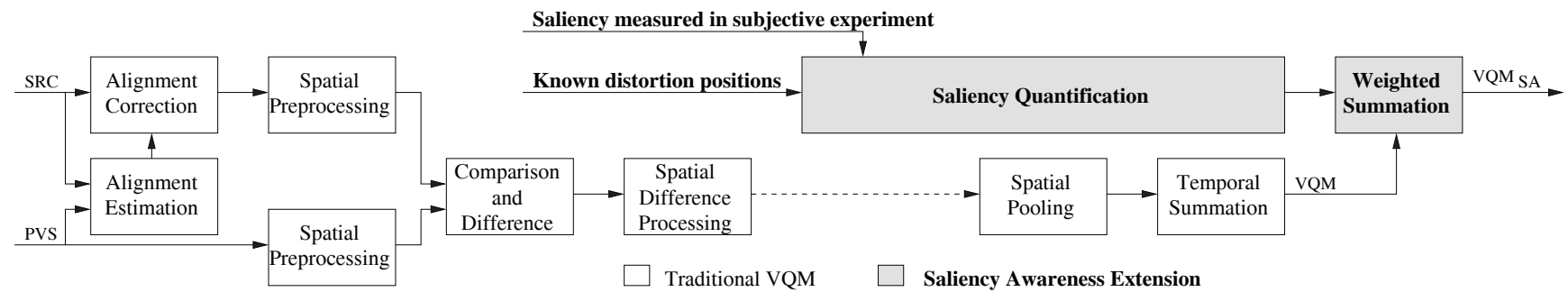

Fig. 5. Overview of the extension of a traditional VQM with the proposed saliency awareness model.

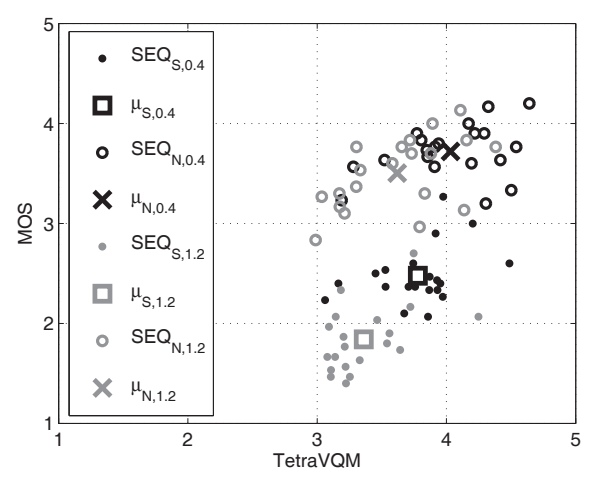

Fig. 4. Scatter plot of TetraVQM versus MOS, highlighting the four subsets of distortions and their mean values.

blocks), without having to change the actual VQM. As can be seen from Fig. 5, the VQM is computed in its regular way and then subjected to a weighted summation with respect to the saliency of the distorted image content as follows

$$
\mathrm{VQM}_{\mathrm{SA}}=\mathrm{VQM}-\alpha \cdot \mathrm{S}
$$

where $\mathrm{S}$ denotes the saliency information. The idea behind Eq. 1 is to add a negative offset $\Delta=-\alpha \cdot \mathrm{S}$ to the $\mathrm{VQM}$ with respect to the amount of saliency information in the region where the distortions appear. This is based on the assumption, that distortions in a more salient region are perceived as more annoying and as such, will receive a lower subjective quality score. In this respect, the parameter $\mathrm{S}$ represents the saliency within the distortion region of a particular video and thus, regulates the relative magnitude of the offset between different contents. The parameter $\alpha$ regulates the general degree with which the offset is performed and needs to be optimised for any particular VQM.

In a practical application, the distorted regions can be detected using distortion maps that are readily available in most traditional VQM. In this work, however, we use the perfect knowledge of the distortion regions due to the controlled creation of the test videos. To avoid potential saliency prediction errors from objective methods, that would subsequently result in errors in the saliency awareness model, we use in this work the saliency maps created from the gaze patterns from the eye tracking experiment (see Section 2.1).
The model outlined here is considered to be generally applicable to any VQM that does not take into account visual saliency. In the following we present two different saliency quantification methods that were found to significantly improve the quality prediction performance of both TetraVQM and PSNR.

\subsection{Saliency quantification method 1}

The saliency awareness model using this first saliency quantification method is in the following referred to as model M1. This method takes into account, that the saliency within the distortion region varies between different videos. For this reason, the saliency in the distorted regions is quantified using the saliency maps created from the gaze patterns. An example of a saliency map, corresponding to the frame presented in Fig. 1, is presented in Fig. 6. The distortion regions are highlighted for the salient region (white grid) and for the nonsalient region (grey grid). The mean saliency is then computed over the whole distortion region as follows

$$
\mathrm{S}_{1}=\frac{1}{\left(\lim _{b}-\lim _{t}\right)\left(\lim _{r}-\lim _{l}\right)} \sum_{m=l i m_{b}}^{\lim _{t}} \sum_{n=l i m_{l}}^{\lim _{r}} \mathrm{~S}(m, n)
$$

where $l i m_{b}, \lim _{t}, \lim _{l}$, and $l i m_{r}$, respectively, denote the limits of the distortion region on the bottom, top, left, and right. The temporal pooling calculates the mean over all degraded frames. The saliency magnitudes $S_{1}$ for all sequences are shown in Fig. 7. One can see that the sequences $\mathrm{SEQ}_{S, 0.4}$ and $\mathrm{SEQ}_{S, 1.2}$ contain a higher amount of saliency as compared to $\mathrm{SEQ}_{N, 0.4}$ and $\mathrm{SEQ}_{N, 1.2}$, however, the amount of saliency is not constant between the different sequences.

\subsection{Saliency quantification method 2}

The saliency awareness model using this saliency quantification method is in the following referred to as model M2. This method does not distinguish between as many saliency levels as M1 does, but rather distinguishes only between two cases; salient region or non-salient region. This can be realised with a threshold algorithm as follows

$$
\begin{array}{lll}
\mathrm{S}_{2}=1 & \text { for } & \mathrm{S}_{1} \geq \tau \\
\mathrm{S}_{2}=0 & \text { for } & \mathrm{S}_{1}<\tau
\end{array}
$$




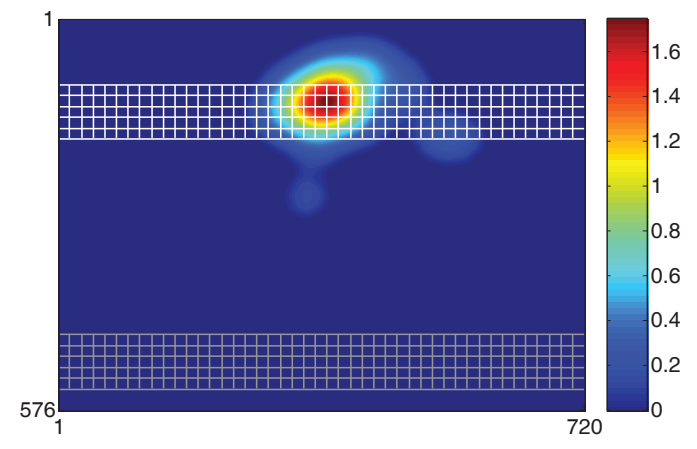

Fig. 6. Saliency map of frame 136 of the video 'Kickoff' with distortion regions highlighted for the salient region (white grid) and the non-salient region (grey grid).

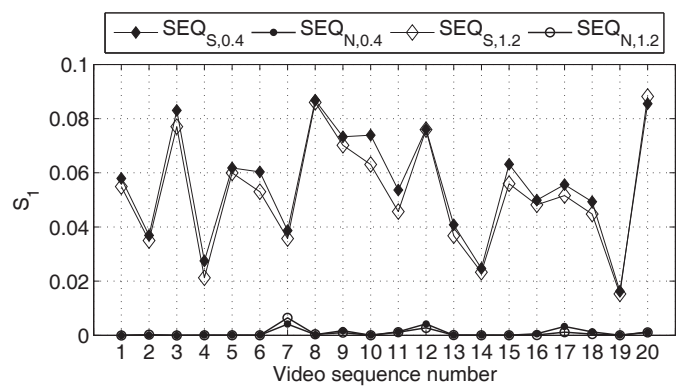

Fig. 7. Saliency quantification $S_{1}$ for all 20 sequence contents in each subset.

Considering the results presented in Fig. 7, we define a threshold of $\tau=0.01$ which separates the classes of saliency and non-saliency in the distorted image content. As such, the VQM scores for the sequences $\mathrm{SEQ}_{S, 0.4}$ and $\mathrm{SEQ}_{S, 1.2}$ receive the same offset, whereas the VQM scores for the sequences $\mathrm{SEQ}_{N, 0.4}$ and $\mathrm{SEQ}_{N, 1.2}$ remain unaltered.

\section{EVALUATION}

The quality prediction performance of the metrics TetraVQM and PSNR is evaluated using three performance indicators; the root mean squared error (RMSE), the Pearson linear correlation coefficient $\varrho_{P}$, and the Spearman rank order correlation $\varrho_{S}$. Prior to calculating the RMSE, a linear fit is applied in order to align the VQM output to the subjective rating scale. For the model enhancements presented in the previous Section, optimal parameters $\alpha_{\text {opt }}$ are determined with respect to minimising RMSE using an exhaustive search. For TetraVQM, the relation between the $\alpha$ and the RMSE is presented in Fig. 8 and the correspondence between $\alpha$ and the correlation coefficients is given in Fig. 9. The minimum RMSE and the maximum $\varrho_{P}$ and $\varrho_{S}$ are highlighted in the respective figures.

The performance values are summarised in Tab. 1 for the VQMs and their proposed enhancements as in TetraVQM ${ }_{M 1}$, TetraVQM 1 2 and PSNR 1 1, PSNR 1 2. The performance results of TetraVQM and PSNR without the proposed en-
Table 1. Optimised parameters $\alpha_{\text {opt }}$ and quality prediction performance indicators for TetraVQM and PSNR.

\begin{tabular}{l|c|ccc}
\hline \hline Metric & $\alpha_{\text {opt }}$ & RMSE & Pearson & Spearman \\
\hline TetraVQM & N/A & 0.702 & 0.522 & 0.536 \\
TetraVQM $_{\mathrm{M} 1}$ & 28.15 & 0.447 & 0.84 & 0.835 \\
TetraVQM $_{\mathrm{M} 2}$ & 2.41 & 0.316 & 0.923 & 0.888 \\
\hline \hline PSNR & N/A & 0.75 & 0.414 & 0.451 \\
PSNR $_{\mathrm{M} 1}$ & 418.61 & 0.465 & 0.825 & 0.83 \\
PSNR $_{\mathrm{M} 2}$ & 35.08 & 0.332 & 0.915 & 0.88 \\
\hline \hline
\end{tabular}

hancements indicate that these metrics are unable to predict the results of this particular subjective experiment consisting of an isolated distortion type. When comparing between TetraVQM and PSNR, it can be observed that TetraVQM consistently performs better than PSNR.

The results show that for both models M1 and M2, the RMSE can be largely decreased and the correlation coefficients can be largely increased. The model M2 achieves better results than model M1, even though M2 does not distinguish saliency levels between the distortion regions of the different sequences, but instead uses a constant offset for $\mathrm{SEQ}_{S, 0.4}$ and $\mathrm{SEQ}_{S, 1.2}$. It should be noted that for $\varrho_{S}$ of model M2, the maximum coincides with the $\alpha_{2}$ for which the TetraVQM $M_{M}$ of all sequences $\mathrm{SEQ}_{S, 0.4}$ and $\mathrm{SEQ}_{S, 1.2}$ are shifted below the TetraVQM $\mathrm{M}_{2}$ of the sequences $\mathrm{SEQ}_{N, 0.4}$ and $\mathrm{SEQ}_{N, 1.2}$. Thus, the rank order correlation of the objective quality scores with MOS is highest when all sequences with distortions in the salient regions are rated lower than the worst sequence with distortions in the non-salient region. This observation is in line with the conclusions drawn from the MOS of the subjective experiment (see Section 2.3 and Fig. 2).

Scatter plots of TetraVQM ${ }_{M 1}$ and TetraVQM ${ }_{M 2}$ are presented in Fig. 10 after deploying a linear mapping to the MOS. The scatter plot for TetraVQM $\mathrm{M}_{2}$ shows two distinct point clouds for the two classes salient and non-salient which partially corresponds to the situation seen in Fig. 2. This is remarkable because the optimization has been performed on the RMSE value and not on the correlation coefficients and thus, it is not an artifact of the training. Nevertheless, it should be noted that the values provided in Tab. 1 provide an upper bound of the expected performance because the same subjective data has been used for training as well as for evaluation.

\section{CONCLUSIONS}

In this paper, we proposed two models of saliency awareness for video quality metrics. The modelling was conducted based on subjective ground truth for both the saliency information and for the annoyance of the packet loss distortions. Application of the models to VQM reveals that the resulting saliency aware metrics show strong improvement in quality 

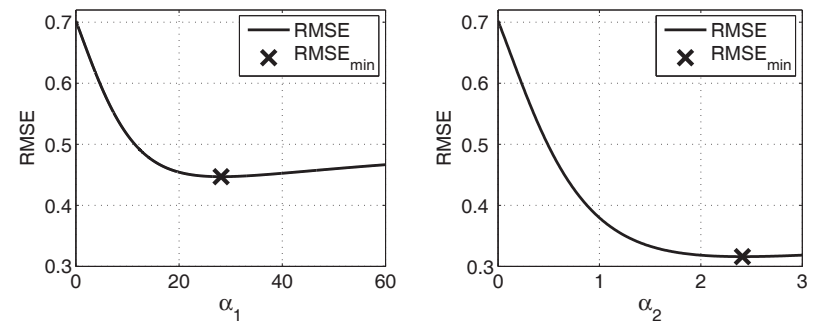

Fig. 8. Root mean squared error (RMSE) versus $\alpha_{1}$ and $\alpha_{2}$.
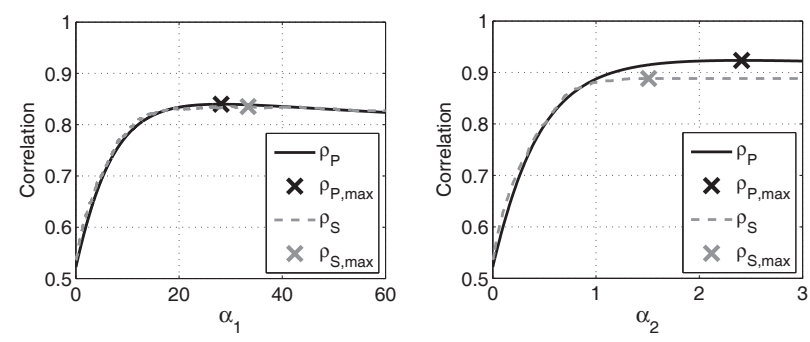

Fig. 9. Pearson correlations $\left(\varrho_{P}\right)$ and Spearman correlations $\left(\varrho_{S}\right)$ versus $\alpha_{1}$ and $\alpha_{2}$.
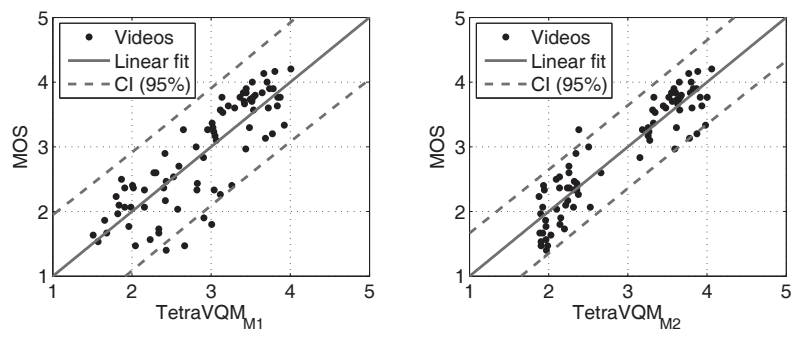

Fig. 10. Scatter plots of TetraVQM $M_{M 1}$ and TetraVQM ${ }_{M 2}$ versus MOS, including 95\% confidence intervals (CI).

prediction performance, as compared to the original metrics.

Further studies are planned to improve the implementation of saliency information in the objective measurement of video quality by incorporating the saliency information directly into the algorithmic processing. This allows for a more comprehensive approach as the measured spatio-temporally localised visual degradations can be weighted immediately with the saliency information.

\section{REFERENCES}

[1] International Telecommunication Union, "Advanced video coding for generic audiovisual services," Rec. H.264, ITU-T, Nov. 2007.

[2] U. Engelke and H.-J. Zepernick, "A framework for optimal region-of-interest based quality assessment in wireless imaging," Journal of Electronic Imaging, Special Section on Image Quality, vol. 19, no. 1, 011005, Jan. 2010.
[3] H. Liu and I. Heynderickx, "Studying the added value of visual attention in objective image quality metrics based on eye movement data," in Proc. of IEEE Int. Conf. on Image Processing, Nov. 2009.

[4] A. K. Moorthy and A. C. Bovik, "Visual importance pooling for image quality assessment," IEEE Journal of Selected Topics in Signal Processing, vol. 3, no. 2, pp. 193-201, Apr. 2009.

[5] A. Ninassi, O. Le Meur, P. Le Callet, and D. Barba, "Does where you gaze on an image affect your perception of quality? applying visual attention to image quality metric," in Proc. of IEEE Int. Conf. on Image Processing, Oct. 2007, vol. 2, pp. 169-172.

[6] L. Itti, C. Koch, and E. Niebur, "A model of saliencybased visual attention for rapid scene analysis," IEEE Trans. on Pattern Analysis and Machine Intelligence, vol. 20, no. 11, pp. 1254-1259, Nov. 1998.

[7] J. You, A. Perkis, M. Hannuksela, and M. Gabbouj, "Perceptual quality assessment based on visual attention analysis," in Proc. of ACM Int. Conference on Multimedia, Oct. 2009, pp. 561-564.

[8] X. Feng, T. Liu, D. Yang, and Y. Wang, "Saliency based objective quality assessment of decoded video affected by packet losses," in Proc. of IEEE Int. Conf. on Image Processing, Oct. 2008, pp. 2560-2563.

[9] M. Barkowsky, J. Bialkowski, B. Eskofier, R. Bitto, and A. Kaup, "Temporal trajectory aware video quality measure," IEEE Journal of Selected Topics in Signal Processing, vol. 3, no. 2, pp. 266-279, Apr. 2009.

[10] NTIA / ITS, “A3: Objective Video Quality Measurement Using a Peak-Signal-to-Noise-Ratio (PSNR) Full Reference Technique," ATIS T1.TR.PP.74-2001, 2001.

[11] U. Engelke, P. Le Callet, and H.-J. Zepernick, "Linking distortion perception and visual saliency in H.264/AVC coded video containing packet loss," in Proc. of SPIE/IEEE Int. Conf. on Visual Communications and Image Processing, July 2010.

[12] Video Quality Experts Group, "VQEG FTP file server," ftp://vqeg.its.bldrdoc.gov/, 2009.

[13] International Telecommunication Union, "Subjective video quality assessment methods for multimedia applications," Rec. P.910, ITU-T, Sept. 1999.

[14] F. Boulos, W. Chen, B. Parrein, and P. Le Callet, "A new H.264/AVC error resilience model based on regions of interest," in Proc. of Int. Packet Video Workshop, May 2009.

[15] Heinrich Hertz Institute Berlin, "H.264/AVC reference software JM 16.1," http://iphome.hhi.de/suehring/tml/, 2009.

[16] Joint Video Team (JVT) of ISO/IEC MPEG and ITU-T VCEG, "SVC/AVC Loss Simulator," http://wftp3.itu.int/av-arch/jvt-site/2005_10_Nice/, 2005.

[17] International Telecommunication Union, "Methodology for the subjective assessment of the quality of television pictures," Rec. BT.500-11, ITU-R, 2002. 